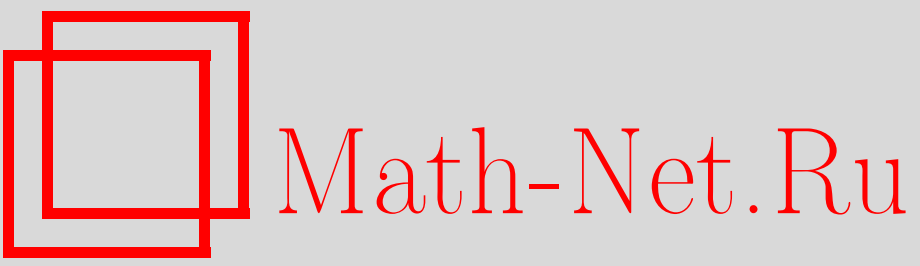

В. А. Юрко, О дискретных операторах высших порядков, УМН, 1996, том 51, выпуск 3, 217-218

DOI: https://doi.org/10.4213/rm992

Использование Общероссийского математического портала Math-Net.Ru подразумевает, что вы прочитали и согласны с пользовательским соглашением

http://www.mathnet.ru/rus/agreement

Параметры загрузки:

IP : 3.95 .254 .165

26 апреля 2023 г., 15:31:21 


\title{
О ДИСКРЕТНЫХ ОПЕРАТОРАХ ВЫСШИХ ПОРЯДКОВ
}

\author{
B. А. Юрко
}

При фиксированных $p, q \geqslant 1$ рассмотрим уравнение

$$
(L y)_{\nu} \equiv \sum_{j=-q}^{p} a_{\nu, j} y_{\nu+j}=\lambda y_{\nu}, \quad \nu \geqslant q, \quad a_{\nu,-q}=1, \quad a_{\nu, p} \neq 0,
$$

где $y=\left[y_{\nu}\right]_{\nu \geqslant 0}, a_{\nu, j}-$ комплексные числа. В статье строится матрица Вейля для (1), исследуются ее свойства и рассматривается обратная задача восстановления несамосопряженного оператора $L$ по матрице Вейля. Получен алгоритм решения обратной задачи, необходимые и достаточные условия ее разрешимости, приведена теорема единственности. Аналогичные результаты справедливы для (1) в абстрактном пространстве. Отметим, что обратная задача для $L$ имеет приложения в теории интегрирования нелинейных эволюционных уравнений (см., например, [1], [2]). Случай $p=q=1$ исследовался в [3]-[6] и других работах. Для дифференциалњных операторов высших порядков обратная задача по матрице Вейля изучалась в [7].

Пусть $\Lambda$ - множество многочленов вида

$$
F(\lambda)=\sum_{k=-j_{1}}^{j_{2}} F_{k} \lambda^{k}
$$

$\left(j_{1}, j_{2} \geqslant 0-\right.$ свои для каждого многочлена). Обозначим $\mathscr{F}$ множество линейных функционалов на $\Lambda$. Элементы $\mathscr{F}$ будем называть обобшенными функциями $(\mathrm{O} \Phi)$. Если $P \in \mathscr{F}$, то числа $P_{k+1}=\left(\lambda^{k}, P\right)$ называются моментами $P$. Здесь $(\cdot, P)$ обозначает действие $P$. Ясно, что ОФ $P \in \mathscr{F}$ своими моментами определяется однозначно по формуле $(F(\lambda), P)=\sum_{k} F_{k} P_{k+1}$, $F(\lambda) \in \Lambda$. ОФ $P \in \mathscr{F}$ удобно записывать в виде формального ряда

$$
P(\lambda)=\sum_{k} P_{k} / \lambda^{k}
$$

ОФ $P \in \mathscr{F}$ можно умножать на элементы $\Lambda$ по формуле $(F(\lambda), G(\lambda) P)=(F(\lambda) G(\lambda), P), F(\lambda)$, $G(\lambda) \in \Lambda$. Это соответствует формальному умножению ряда $(2)$ на $G(\lambda)$. Через $\mathscr{F}^{+}$обозначим множество ОФ $P \in \mathscr{F}$, для которых $P_{k}=0$ при $k<0$, а через $\mathscr{F}_{0}^{+}-$множество ОФ $P \in \mathscr{F}^{+}$, для которых $P_{0}=0$.

Пусть $\Phi_{\nu}=\left[\Phi_{\nu}^{i}(\lambda)\right]_{i=\overline{1, q}}^{T}, \nu \geqslant 0,-$ решение уравнения (1) при условиях

$$
\Phi_{\nu}^{i}(\lambda)=\sum_{k=0}^{\infty} \frac{1}{\lambda^{k}} \Phi_{k \nu}^{i} \in \mathscr{F}^{+}, \quad \Phi_{\nu}^{i}(\lambda)=\delta_{i, \nu+1} \quad(\nu=\overline{0, q-1}) .
$$

Здесь $\delta_{i j}$ - символ Кронекера, а $T$ - знак транспонирования (т.е. $\left[a_{i}\right]-$ столбец, $\left[a_{i}\right]^{T}-$ строка). Введем матрицу $\mathfrak{M}(\lambda)=\left[\mathfrak{M}_{j}^{i}(\lambda)\right]_{j=\overline{1, p} ; i=\overline{1, q}}$ по формуле $\mathfrak{M}_{j}^{i}(\lambda)=\Phi_{j+q-1}^{i}(\lambda)$. Матрицу $\mathfrak{M}(\lambda)$ будем называть матрицей Вейля оператора $L$.

Обратная задача ставится следующим образом: по заданной матрице Вейля $\mathfrak{M}(\lambda)$ построить оператор $L$.

Справедлива следующая теорема единственности.

Teорема 1. Пусть $\mathfrak{M}(\lambda), \mathfrak{M}^{0}(\lambda)$ - матрицъ Вейля для операторов $L$ u $L^{0}$, coomветственно. Тогда, если $\mathfrak{M}(\lambda)=\mathfrak{M}^{0}(\lambda)$, то $L=L^{0}$.

Обозначим $R_{k}(\lambda)=\left[R_{k}^{j}(\lambda)\right]_{j=\overline{1, p}}^{T}, R_{p k+i-1}^{j}(\lambda)=\delta_{i j} \lambda^{k}, i=\overline{1, p} ; R_{k}^{*}(\lambda)=\left[R_{k}^{*, j}(\lambda)\right]_{j=\overline{1, q}}$, $R_{k q+i-1}^{*, j}(\lambda)=\delta_{i j} \cdot \lambda^{k}, i=\overline{1, q}$. Пусть $P_{k}(\lambda)=\left[P_{k}^{j}(\lambda)\right]_{j=\overline{1, p}}^{T}, Q_{k}(\lambda)=\left[Q_{k}^{i}(\lambda)\right]_{i=\overline{1, q}}^{T}, k \geqslant 0$,

Работа выполнена при поддержке ГК РФ по высшему образованию (грант № 96-1.7-4). 
- решения уравнения (1) при начальных условиях $P_{k}(\lambda)=0(k=\overline{0, q-1}), P_{k+q}(\lambda)=R_{k}(\lambda)$ $(k=\overline{0, p-1}) ; Q_{k}^{i}(\lambda)=\delta_{k+1, i}(k=\overline{0, q-1}) ; Q_{k+q}(\lambda)=0(k=\overline{0, p-1})$. Тогда

$$
P_{k+q}(\lambda)=\sum_{i=0}^{k} c_{i k} R_{i}(\lambda), \quad k \geqslant 0, \quad c_{k k} \neq 0
$$

Подставляя (3) в (1) и приравнивая коэффициенты при $R_{i}(\lambda)$, находим

$$
\sum_{j=-q}^{p} a_{k j} c_{i, k+j-q}=c_{i-p, k-q}, \quad i=\overline{0, k+p-q}
$$

Отсюда получаем рекуррентные формулы для определения коэффициентов $a_{k \nu}$ через $c_{i k}$ :

(4) $a_{k \nu}=\left(c_{k+\nu-q, k+\nu-q}\right)^{-1}\left(c_{k+\nu-q, k-q}-\sum_{j=\nu+1}^{p} a_{k j} c_{k+\nu-q, k+j-q}\right), \quad \nu=\overline{p,-q+1}$.

Здесь $k \geqslant q$ при $\nu \geqslant 0 ; k \geqslant q-\nu$ при $\nu<0$ и доопределено $c_{i k}=0$ при $i<0$. В частности, $a_{k p}=\left(c_{k+p-q, k+p-q}\right)^{-1} \cdot c_{k-q, k-q}$.

Лемма 1. Справедливы следующие соотношения: $\Phi_{k}(\lambda)=Q_{k}(\lambda)+P_{k}(\lambda) \mathfrak{M}(\lambda), k \geqslant 0$,

$$
\begin{array}{lll}
\left(1, P_{k+q}(\lambda) \mathfrak{M}(\lambda) R_{\nu}^{*}(\lambda)\right)=a_{q+k, \nu-q-k}, & & 0 \leqslant k<\nu \leqslant q-1, \\
\left(1, P_{k+q}(\lambda) \mathfrak{M}(\lambda) R_{\nu}^{*}(\lambda)\right)=\delta_{\nu k}, & & 0 \leqslant \nu \leqslant k .
\end{array}
$$

Обозначим $\mu_{i \nu}=\left(1, R_{i}(\lambda) \mathfrak{M}(\lambda) R_{\nu}^{*}(\lambda)\right), i, \nu \geqslant 0 ; \Delta_{k}=\operatorname{det}\left[\mu_{i \nu}\right]_{i, \nu=\overline{0, k}}, k \geqslant 0$. Так как $P_{k+q}(\lambda)=R_{k}(\lambda)$ при $k=\overline{0, p-1}$, то $\mu_{i \nu}=\delta_{i \nu}$ при $0 \leqslant \nu \leqslant i \leqslant p-1$. Подставляя (3) в (6), получим

$$
\sum_{i=0}^{k} c_{i k} \mu_{i \nu}=\delta_{\nu k}, \quad 0 \leqslant \nu \leqslant k
$$

При фиксированном $k \geqslant 0(7)$ - линейная алгебраическая система с определителем $\Delta_{k}$.

Лемма 2. При всех $k \geqslant 0 \quad \Delta_{k} \neq 0$.

Решая системы $(7)$, находим

$$
c_{i k}=(-1)^{k-i} \cdot \Delta_{k}^{-1} \cdot \operatorname{det}\left[\mu_{j \nu}\right]_{j=\overline{0, k} \backslash i ; \nu=\overline{0, k-1}}, \quad c_{k k}=\Delta_{k-1} \cdot \Delta_{k}^{-1} .
$$

Пусть $M$ - множество матрищ $\mathfrak{M}(\lambda)=\left[\mathfrak{M}_{j}^{i}(\lambda)\right]_{j=\overline{1, p}}, i=\overline{1, q}, \mathfrak{M}_{j}^{i}(\lambda) \in \mathscr{F}_{0}^{+}$, таких, что $\mu_{i \nu}=$ $\delta_{i \nu}$ при $0 \leqslant \nu \leqslant i \leqslant p-1$. Следуюшая теорема дает необходимые и достаточные условия разрешимости обратной задачи и алгоритм ее решения.

Теорема 2. Для того, чтобъ матрица $\mathfrak{M}(\lambda) \in M$ бъла матрицей Вейля для некоторого оператора вида (1), необходимо и достаточно, чтобы $\Delta_{k} \neq 0$ при всех $k \geqslant 0$. При әтом оператор L находится по следующему алгоритму.

Алгоритм 1. (1) Строим $c_{i k}(0 \leqslant i \leqslant k) u P_{k+q}(\lambda)$ nри $k \geqslant 0$ по формулам (8) $и$ (3) u полагаем $P_{k}(\lambda)=0$ при $k=\overline{0, q-1}$. (2) Вычисляем $a_{k \nu}$ по формулам (4), (5).

СлеДСТвиЕ 1. Для того, чтобъ матрица $\mathfrak{M}(\lambda) \in M$ была матрицей Вейля для некоторого оператора $L$ с вещественными коэффичиентами $a_{\nu j}$ и $a_{\nu p}>0$, необходимо и достаточно, чтобь числа $\mu_{i \nu}$ были вещественными $u \Delta_{k}>0$ при всех $k \geqslant 0$.

\section{СПИСОК ЛИТЕРАТУРЫ}

[1] Березанский Ю. М. // ДАН СССР. 1985. Т. 281. №1. С. 16-19. [2] Богоявленский О. И. // Изв. АН СССР. Сер. матем. 1987. Т. 51. №6. С. 1123-1141. [3] Аткинсон Ф. Дискретные и непрерывные граничные задачи. М.: Мир, 1968. [4] Березанский Ю. М. Разложение по собственным функциям самосопряженных операторов. Киев, 1965. [5] Ахиезер Н. И. Классическая проблема моментов. М., 1961. [6] Гусейнов Г. Ш. // Матем. заметки. 1978. Т. 23. № 2. С. 237-248. [7] Юрко В. А. // Матем. сб. 1991. Т. 182. № 3. С. 431-456. 